\title{
MeerKAT Configuration Studies
}

\author{
Anja C. Schröder ${ }^{* a b}$, B. Frank ${ }^{c}$, W.J.G. de Blok ${ }^{c}$, J. Jonas ${ }^{d}$ and M. de Villiers, ${ }^{b}$ \\ ${ }^{a}$ Hartebeesthoek Radio Astronomy Observatory, PO Box 443, Krugersdorp 1740, South Africa \\ ${ }^{b}$ SKA/KAT, Lonsdale Building, Lonsdale Road, Pinelands 7405, Cape Town, South Africa \\ ${ }^{c}$ Department of Astronomy, University of Cape Town, Private Bag X3, Rondebosch 7701, South \\ Africa \\ ${ }^{d}$ Department of Physics $\mathcal{E}$ Electronics, Rhodes University, Grahamstown 6140, South Africa \\ E-mail: anja@hartrao.ac.za,
}

\begin{abstract}
The evolution of the HI content in the universe is one of the key science drivers for the building of the SKA precursor instrument MeerKAT. It is therefore important that the antenna configuration allows sensitivity to low surface brightness structures. On the other hand, sensitivity at a high resolution is desired by continuum studies and transient sciences. The highest sensitivity, however, can be achieved only for an array optimized for one specific resolution. We present here the evaluation process to arrive at an array configuration optimal for a wide range of resolutions.
\end{abstract}

Panoramic Radio Astronomy: Wide-field 1-2 GHz research on galaxy evolution

June 2-5 2009

Groningen, the Netherlands

\footnotetext{
* Speaker.
} 


\section{Introduction}

The Karoo Array Telescope, MeerKAT, is South Africa's SKA precursor and will most likely consist of 80 dishes with a $12 \mathrm{~m}$ diameter. The dual-polarisation single-pixel receivers will have a desired $T_{\text {sys }}$ of $30 \mathrm{~K}$. The array will be located near the proposed SKA core site in the Karoo region of the Northern Cape Province. Key sciences include the evolution of galaxies and large-scale structures, dark matter, cosmic magnetism and the nature of transient radio sources. Table 1 lists the dependence of resolution on maximum baselines in the array and the pros and cons for the key science cases.

Table 1: Science cases for various resolution regimes

\begin{tabular}{llll}
\hline Resolution regime & Configuration & Ideal for: & Less suited for: \\
\hline $\begin{array}{l}\text { Low resolution: } \\
\left(<\sim 1^{\prime}\right)\end{array}$ & $\begin{array}{l}\text { mainly short } \\
\text { baselines }\end{array}$ & $\begin{array}{l}\text { - high column sensitivity } \\
\text { - mapping extended low column density }\end{array}$ & - galaxy surveys \\
& & structures (cosmic web, outskirts of nearby galaxies $)$ & detailed analyses of the ISM \\
Medium resolution: & mainly medium & - galaxy surveys & - tracing low densities \\
$\left(\sim 20^{\prime \prime}-30^{\prime \prime}\right)$ & long baselines & - limited mapping of nearby galaxies & - high column densities \\
High resolution: & mainly very & - detailed mapping of galaxies and their environment & \\
$\left(\sim 5^{\prime \prime}-10^{\prime \prime}\right)$ & long baselines & - studies of evolution of the ISM in galaxies & \\
& & - detailed studies of kinematics of galaxies & \\
\hline
\end{tabular}

\section{Single-resolution array}

In several steps, we investigated the effect of various parameters on the performance of a single-resolution Gaussian array (characterised by the baseline distribution, $\sigma$, and maximum baseline). We investigated three sets of parameters, $(\sigma, \max )=(200 \mathrm{~m}, 750 \mathrm{~m}),(700 \mathrm{~m}, 2500 \mathrm{~m})$ and $(2500 \mathrm{~m}, 8000 \mathrm{~m})$, but we show the results for $(700 \mathrm{~m}, 2500 \mathrm{~m})$ only, with an angular resolution $\simeq 24^{\prime \prime}$ using natural weighting, since it is representative for arrays optimised for other resolutions.

(i) We optimised the array for a range of observing times $t$ and declinations $\delta$ and evaluated the PSF residuals. Figure 1 shows the maximum values of positive and negative residuals for various $\delta$ and $t$. As the positive residuals are dominating, the right panel shows only the maximum positive residuals in a different display. While a large range of $\delta$ and $t$ values show small residuals, the worst case is (as expected) $\delta=+10^{\circ}$. We choose the $(\delta, t)=\left(-70^{\circ}, 8 \mathrm{~h}\right)$ array as the reference array for the following evaluations (though the conclusions are largely independent on the exact choice).

(ii) As a first test, we evaluated the chosen array for other $(\delta, t)$ combinations, and the PSF residuals are shown in Fig. 2. The performance is satisfactorily for most sets of $(\delta, t)$ except for long observations at equatorial declinations (this is expected since the range in hour angle is limited here which results in relatively poor beam shapes).

(iii) To evaluate the chosen array for performance in the other two resolution regimes, we used the weighting method by one of us (M. de Villiers, see the package AntConfigServer) to weight the individual ungridded uv-samples in such a way that a beam is produced with the desired resolution and which is as close to a Gaussian as possible (note that this depends on the availability in the original array of a sufficient number of baselines corresponding to the desired resolution). Table 2 lists for each combination of optimum and target $\sigma$ the sensitivity as a fraction 

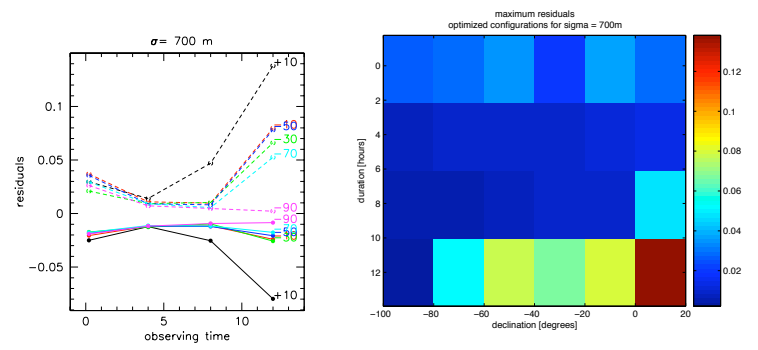

Figure 1: Gaussian arrays optimised for different declination and observing times.
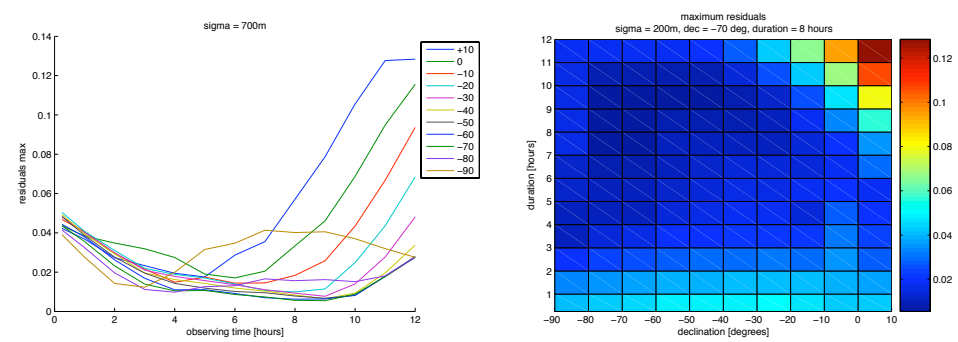

Figure 2: Behaviours of Gaussian arrays at non-optimised declination and observing times.

of natural weighted sensitivity of an optimised Gaussian array at that resolution (first number) and the maximum residual in percent (second number).

Our conclusion is that for arrays optimised for a single resolution one can use the weighting to "tune" to different resolutions but only at a great cost in sensitivity.

Table 2: Fractional sensitivities and residuals for Gaussian arrays

\begin{tabular}{lccc}
\hline & $\sigma_{\text {target }}=200 \mathrm{~m}$ & $\sigma_{\text {target }}=700 \mathrm{~m}$ & $\sigma_{\text {target }}=2500 \mathrm{~m}$ \\
\hline$\sigma_{\text {optim }}=200 \mathrm{~m}$ & $1.00 / 0.4 \%$ & $0.04 / 31.6 \%$ & $0.02 / 85.0 \%$ \\
$\sigma_{\text {optim }}=700 \mathrm{~m}$ & $0.34 / 0.1 \%$ & $1.00 / 0.6 \%$ & $0.04 / 32.9 \%$ \\
$\sigma_{\text {optim }}=2500 \mathrm{~m}$ & $0.07 / 3.3 \%$ & $0.33 / 0.4 \%$ & $1.00 / 0.7 \%$ \\
\hline
\end{tabular}

\section{Multi-resolution array}

As we have seen, Gaussian arrays have a poor sensitivity at other resolutions than the one optimised for. To configure an array capable of multiple resolutions, we used weighting of the uv-samples to "tune" to the desired resolution and minimise the PSF residuals. As a consequence, such arrays have a sensitivity less than that of an optimised array at the desired resolution. In our studies we gave highest priorities to minimising sensitivity loss for the medium and high resolution cases as required by the majority of $\mathrm{H}$ I science cases. We have investigated two types of arrays:

(i) A hybrid array: an inner Gaussian core optimised for $\sigma=700 \mathrm{~m}$ plus an outer Gaussian distribution with $\sigma=2500 \mathrm{~m}$. The ratio of the number of antennae in each component was initially set to be $70 \% / 30 \%$ and $50 \% / 50 \%$.

(ii) A pinched Gaussian array, i.e., a Gaussian array, optimised for $\sigma=2500 \mathrm{~m}$, where subsequently the distance from the centre of the array $d$ of each antenna was multiplied by $\left(d / d_{\max }\right) \gamma$, 


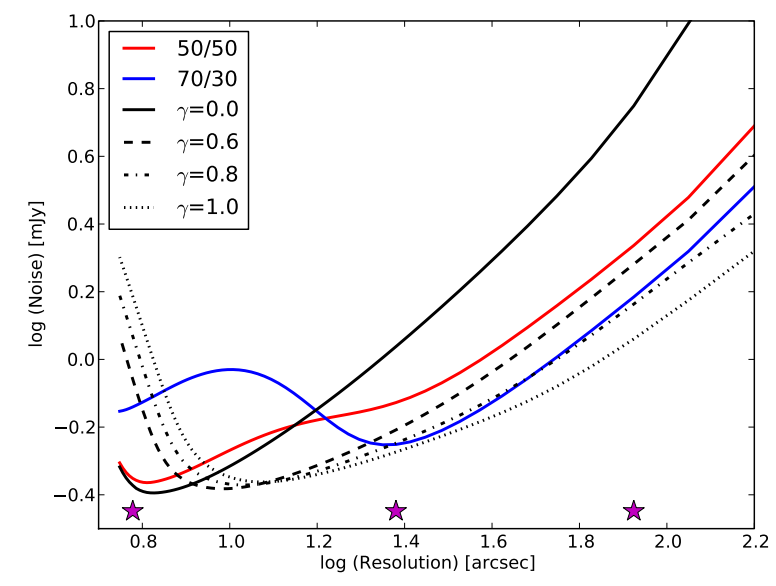

Figure 3: Comparison of hybrid and pinched arrays. Low noise is equivalent to high sensitivity.

where $d_{\max }$ is the maximum antenna distance from the centre of the array, and $\gamma$ is the pinch parameter with a value between 0 and 1 . The pinching is used to give larger emphasis to short baselines and results in an enhanced sensitivity to low column densities.

Figure 3 shows the comparison of the two hybrid arrays and the pinched array with various $\gamma$ values (see legend in the figure). It is obvious that the hybrid arrays are significantly worse than the pinched array at intermediate resolutions. Note that none of these arrays were optimised for resolutions above 1 arcminute. The value of $\gamma$ determines the trade-off between sensitivities at the high versus the low resolution end. Each of the three stars indicates the best sensitivity that can be achieved by fully optimising an array for each of the single resolutions mentioned above, respectively. However, at resolutions away from the ideal (i.e., location of the star) the sensitivities are significantly worse (not shown).

\section{Final MeerKAT configuration}

The final configuration was revisited after the conference and is up-dated here. The main goal for the MeerKAT array configuration is a roughly constant point source sensitivity for resolutions $\sim 8^{\prime \prime} \sim 100^{\prime \prime}$. The array will consist of a $70 \% / 30 \%$ hybrid Gaussian array with $(\sigma, \max )=(300$, $1000 \mathrm{~m}$ ) and $(2500 \mathrm{~m}, 8000 \mathrm{~m})$, respectively (see de Blok, 2009, for details). Such an array is fairly sensitive to the ratio of number of antennae in each component, and the chosen ratio gives a smooth transition between high and low resolution regimes. A 'pinching' solution has been dropped, and a recent improvement in the weighting scheme (de Villiers, in prep.) will slightly enhance the sensitivity performance over the one shown above, especially at lower resolutions. To fulfil the requirement for high resolution by continuum studies and transient science, the seven antennae from the prototype KAT-7 may be placed along the access road to the MeerKAT site which goes for $\sim 50 \mathrm{~km}$ roughly in an East-West direction between Carnarvon and the MeerKAT site.

\section{References}

[1] de Blok, W.J.G. 2009, these proceedings 